\title{
Comparison of subclinical dermatophyte infection in short- and long-haired cats
}

\author{
Panpicha Sattasathuchana ${ }^{1}$, Chunyaput Bumrungpun² (i) and Naris Thengchaisri ${ }^{1}$ (D) \\ 1. Department of Companion Animal Clinical Sciences, Kasetsart University, Bangkok 10900, Thailand; 2. The Veterinary \\ Diagnosis Laboratories, Faculty of Veterinary Medicine, Kasetsart University, Bangkok, 10900, Thailand. \\ Corresponding author: Naris Thengchaisri, e-mail: ajnaris@yahoo.com \\ Co-authors: PS: psatta99@gmail.com,CB: fvetcpb@ku.ac.th \\ Received: 24-07-2020, Accepted: 17-11-2020, Published online: 29-12-2020
}

doi: www.doi.org/10.14202/vetworld.2020.2798-2805 How to cite this article: Sattasathuchana P, Bumrungpun C, Thengchaisri N (2020) Comparison of subclinical dermatophyte infection in short- and long-haired cats, Veterinary World, 13(12): 2798-2805.

\begin{abstract}
Background and Aim: Long-haired cats may have an increased risk of dermatophytosis due to insufficient grooming and their thick hair coat trapping fungal spores. The prevalence of subclinical dermatophytosis in long-haired cats was evaluated using fungal culture and Wood's lamp test. Hematology and blood chemistry results were compared between cats negative and positive for dermatophytosis.
\end{abstract}

Materials and Methods: A total of 127 cats (median age, 3 years [range, 10 months-10 years]) without feline leukemia virus or feline immunodeficiency virus infection were classified into short-haired $(n=64)$ and long-haired ( $n=63)$ groups. Hair samples were cultured on a fungal culture medium (dermatophyte test medium, enhanced sporulation agar, and Sabouraud agar).

Results: The prevalence of dermatophytosis in short-haired and long-haired cats was $6.25 \%(95 \%$ confidence interval [CI], 2.15-12.28) and 34.92\% (95\% CI, 22.94-46.90), respectively. The odds of long-haired cats having dermatophytosis were 8.05 (95\% CI, 2.44-33.97) times greater than that in short-haired cats. The number of positive dermatophytosis found in domestic short-haired cats $(2 / 50,4.0 \%)$ was significantly lower than that in Persian cats $(17 / 47,36.17 \% ; p<0.001)$ and long-haired mixed breed cats $(3 / 7,42.86 \% ; \mathrm{p}=0.011)$. The overall sensitivity and specificity of the Wood's lamp test for diagnosing Microsporum canis infection were 37.5\% (95\% CI, 21.2-57.3\%) and 96.1\% (95\% CI, 90.4-98.5\%), respectively. Cats with dermatophytosis had significantly lower hematocrit and serum albumin levels than cats without dermatophytosis.

Conclusion: Subclinical dermatophytosis was more common in long-haired cats; therefore, dermatophyte examinations should be performed routinely.

Keywords: cats, dermatophyte, hematology, mycosis, Wood's lamp.

\section{Introduction}

Dermatophytosis is a common and highly contagious zoonotic disease seen in cats [1,2]. Microsporum canis is the most commonly isolated pathogen in feline dermatophytosis [2]. Microsporum persicolor, Microsporum gypseum, Trichophyton verrucosum, Trichophyton mentagrophytes, and Epidermophyton species have been isolated in a few cats [2-6]. Diagnostic methods, such as skin cytology, biopsy, polymerase chain reaction, and fungal cultures, have been used to detect dermatophyte infection [1,3,6-13]. Although Wood's lamp is the fastest dermatophytosis test, it has a low sensitivity [3]. Fungal culture provides highly specific test results for detecting dermatophytosis, but it is time-consuming $[1,12]$. Numerous testing media have been used to isolate and identify dermatophyte species [12]. Dermatophyte test medium

Copyright: Sattasathuchana, et al. Open Access. This article is distributed under the terms of the Creative Commons Attribution 4.0 International License (http://creativecommons.org/licenses/ by/4.0/), which permits unrestricted use, distribution, and reproduction in any medium, provided you give appropriate credit to the original author(s) and the source, provide a link to the Creative Commons license, and indicate if changes were made. The Creative Commons Public Domain Dedication waiver (http:// creativecommons.org/publicdomain/zero/1.0/) applies to the data made available in this article, unless otherwise stated.
(DTM) and enhanced sporulation agar (ESA) are selective dermatophyte test media containing dermatophyte growth enhancers and dye indicators [1,14,15], and Sabouraud dextrose agar (SDA) is a non-specific, classic universal agar that is used to grow saprophytes [1]. Dermatophytes quickly grow on SDA [12], and a combination of DTM, ESA, and SDA increases the ability to detect dermatophytosis and other fungal diseases.

Dermatophytes are not normal fungal flora of cats [16]. Isolation of these fungal species in healthy animals indicates subclinical infection and asymptomatic transient carriage $[2,17,18]$. Important factors related to dermatophyte infection include age, lifestyle, living situation (indoor vs. outdoor), and climate of its living area (outdoor cats living in warmer climates are more susceptible to dermatophytosis) [1]. Cats of all ages can contract the disease, but kittens are commonly affected $[1,5]$. It is still unclear whether cats that are infected with feline leukemia virus (FeLV) or feline immunodeficiency virus (FIV) are more susceptible to dermatophyte infection $[10,17,18]$. Although long-haired cats have a long and thick coat in which fungal spores may become trapped, the relationship between coat length and infection with dermatophytes remains unclear. 
The present study aimed to: (1) Identify and compare the prevalence of dermatophytosis in shortand long-haired cats without FeLV and FIV infection; (2) determine the association between dermatophytosis and hair length; (3) evaluate the sensitivity, specificity, positive predictive value, and negative predictive value of the Wood's lamp test for dermatophytosis detection; and (4) compare hematology and blood chemistry parameters of cats with and without dermatophytosis.

\section{Materials and Methods}

\section{Ethical approval and informed consent}

The sample collection protocols were reviewed and approved by the Animal Care and Use Committee at Kasetsart University (ACKU60-VET-032). The informed owner consent forms were signed before samples were collected.

\section{Study period and location}

The samples were collected from cats visiting Kasetsart University Veterinary Teaching Hospital, Bangkok, Thailand, for routine health check between July 2017 to June 2018.

\section{Animals}

A total of 127 privately owned indoor cats were enrolled in the hematology and dermatophytosis survey. The age, sex, breed, and hair length of the cats were recorded. The cats were divided into two groups: Short-haired and long-haired cats. Cats with a hair shaft length $<3 \mathrm{~cm}$ were classified as the short-haired cat group and the other cats were classified as the long-haired cat group. A general physical examination was performed on all cats. None of the cats showed dermatological signs. Approximately $3 \mathrm{~mL}$ of blood was collected through the jugular vein and transferred to ethylenediaminetetraacetic acid-coated tubes and plain glass tubes for complete blood count and blood chemistry testing, respectively. Complete blood count was performed using an automated hematology analyzer (Abbott CELL-DYN 3700 Hematology Analyzer, Abbott, Germany) to measure hematocrit, white blood cell count, neutrophil count, lymphocyte counts, monocyte count, and eosinophil count. Blood chemistry was performed using a laboratory chemical analyzer (ILab650 Automatic Biochemistry analyzer, Instrumental Laboratory, USA) to measure blood urea nitrogen, creatinine, alanine aminotransferase, aspartate aminotransferase, alkaline phosphatase, total protein, albumin, and globulin. Cats with confirmed positive results for FeLV or FIV using rapid immunochromatographic tests (FASTest FeLVFIV, MEGACOR Diagnostik GmbH, Austria) were excluded from the study.

\section{Wood's lamp skin examination}

All cats were tested for M. canis using Wood's lamp test in a dark room. A glowing hair shaft with a bright apple green to yellowish color was considered a positive result (Figure-1). Both fluorescent colors of scale and non-fluorescent color were considered a negative test result.

\section{Fungal culture}

Hair and scale samples were collected for fungal culture using Mackenzie's toothbrush technique in which a sterile toothbrush was used to brush the hair coat for 20 strokes or at least $2 \mathrm{~min}$ [1]. Hair shafts and scales were transferred to a combi culture agar plate containing three separate media (DTM, ESA, and SDA; MYKODERMOASSAY Trio, MEGACOR Diagnostik $\mathrm{GmbH}$, Austria). Agar plates were then incubated at room temperature $\left(25^{\circ} \mathrm{C}-30^{\circ} \mathrm{C}\right)$. The DTM and ESA culture agars were closely monitored for color changes. Dermatophyte colonies were identified by a change in the orange color of the DTM agar and the yellow color of the ESA agar to red and greenblue, respectively (Figure-2). Colony growth on the DTM, ESA, and SDA culture agars was monitored for 21 days [12]. Microscopic examination of wooly, fluffy colonies was performed on all positive agars to identify the fungal hyphae and spores, as shown in Figure-3. All fungal species were recorded. After 21 days, any non-growing colonies and media showing no color change were discarded and reported as negative for fungi.

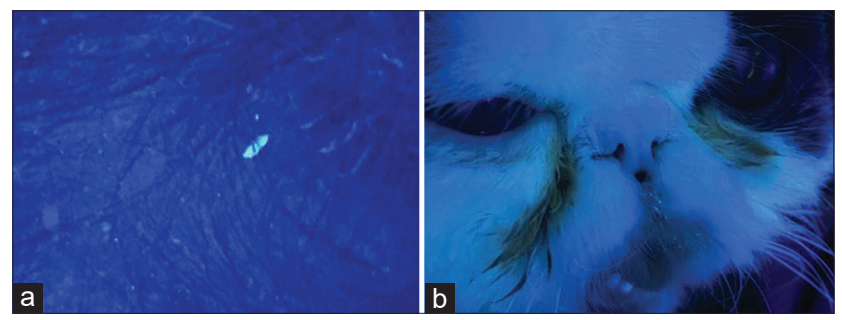

Figure-1: The images of the positive Wood's lamp test of short-haired and long-haired cats. (a) A bright green fluorescent spot was detected on the base of a hair shaft over the truncal area in a short-haired cat with positive to Microsporum canis. (b) Green flurescencing facial hairs were identified in a long-haired cat with positive to M. canis.

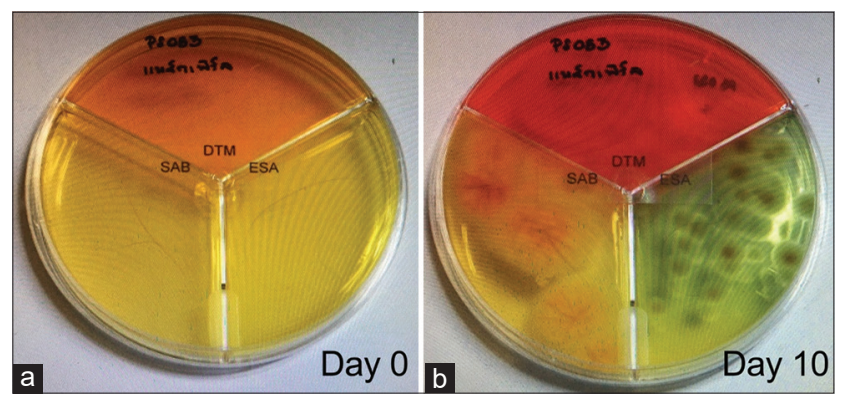

Figure-2: The MYCODERMOASSAY Trio was used for rapid detection of dermatophyte. (a) Hair samples (day 0) from each cat were cultured on the MYCODERMOASSAY Trio. (b) Early diagnosis can be made by detecting the color changes on the fungal plate (day 10). The characteristic of dermatophyte colony can be found in Sabouraud (SAB) agar. Color changes from orange to red can be detected in the dermatophyte test medium for positive dermatophyte colony. Color changes from yellow to green can be detected in the enhanced sporulation agar (ESA) for positive dermatophyte colony. Microscopic differentiation of dermatophytes can be visualized using colony from the ESA. 


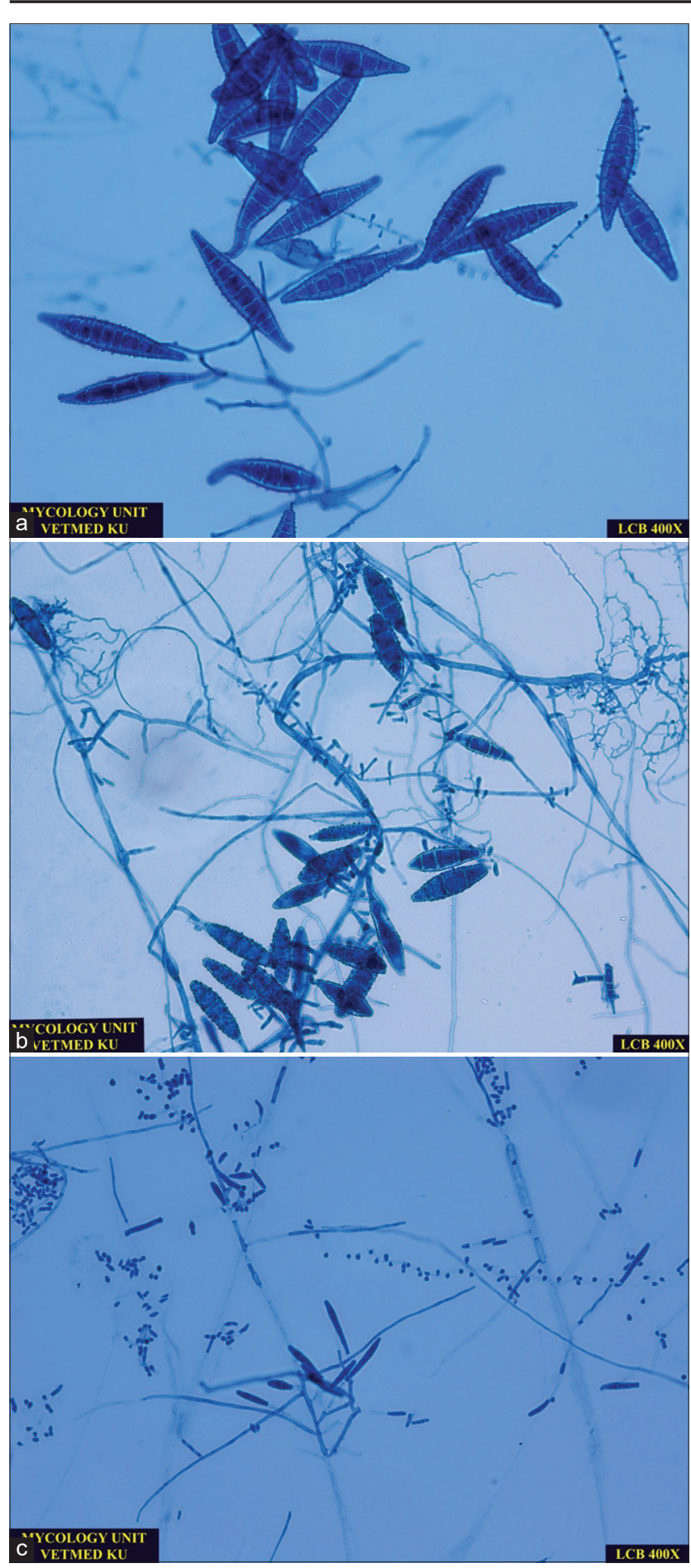

Figure-3: Microscopic images of common dermatophyters identified in cats using the lactophenol cotton blue staining. (a) Microsporum canis is confirmed by a presence of spindle-shaped, rough, thick-walled, macroconidia, and few round-shaped microconidia $(400 \times)$. (b) M. gypseum is confirmed by a presence of boat-shaped, rough, thinwalled macroconidia and few club-shaped microconidia $(400 \times)$. C: Trichophyton mentagrophytes is confirmed by a presence of cigar-shaped, smooth, thin-walled macroconidia and abundant grape-like clusters of microconidia (400x).

\section{Statistical analysis}

Statistical analyses were performed using commercially available statistical software packages (JMP Pro 10, SAS Institute, Cary, North Carolina, USA; GraphPad Prism version 6.0, GraphPad Software, Inc.,
La Jolla, California, USA; STATA v 12, StataCorp, College Station, Texas, USA). The significance level was set at $\mathrm{p}<0.05$. Shapiro-Wilk $\mathrm{W}$ test was used to assess the normality of the data. Student's t-test was performed to compare the age and body weight of the cats. Comparison of the categorical variables, including sex, was performed using the Pearson $\chi^{2}$ test.

Positive test results from the dermatophyte cultures were used to calculate the prevalence and the $95 \%$ confidence interval (CI) of dermatophytosis in the long- and short-haired cats. The association between hair length and each type of fungal species was determined using Fisher's exact test, and the odds ratio of dermatophytosis in long-haired cats also was calculated. The sensitivity, specificity, positive predictive value, negative predictive value, and CI of the Wood's lamp for diagnosing $M$. canis infection also were determined. Hematology and blood chemistry parameters were compared between cats with dermatophytosis and cats without dermatophyte infection. Student's t-test and Wilcoxon rank-sum test were selected to apply on parametric data and non-parametric data, respectively. The significance level was set at $\mathrm{p}<0.05$.

\section{Results}

The demographic characteristics of the cats, including age $(p=0.8847)$ and sex $(p=0.1106)$, were not significantly different between the short- and long-haired groups (Table-1). However, the shorthaired cats weighed more than the long-haired cats $(\mathrm{p}=0.0131)$.

Dermatophyte infection was identified in 26 cats (Table-2), with Microsporum and Trichophyton species isolated in 24 and 2 cats, respectively. Other fungi were also identified, including Absidia species ( $\mathrm{n}=8)$, Aspergillus species $(\mathrm{n}=43)$, Bipolaris species $(\mathrm{n}=5)$, Chaetonium species $(\mathrm{n}=2)$, Cladophialophora species $(\mathrm{n}=2)$, Cladosporium species $(\mathrm{n}=12)$, Curvularia species $(\mathrm{n}=5)$, Nigrospora species $(\mathrm{n}=2)$, Paecilomyces species $(\mathrm{n}=3)$, Penicillium species $(\mathrm{n}=12)$, Rhizopus species $(n=4)$, and Trichoderma species $(n=3)$. There was a significant association between hair length and dermatophytosis $(\mathrm{p}<0.0001)$. Associations between other fungal species and hair length are shown in Table-2.

There was significantly more positive Wood's lamp test results seen in long-haired cats $(10 / 63$, $15.87 \%)$ compared with short-haired cats (3/64, $4.69 \% ; p=0.0330$ ) (Table-3). Images of the positive Wood's lamp test results for short- and long-haired cats are shown in Figure-1. The prevalence of dermatophytosis in long-haired cats $(22 / 63,34.92 \%$ [95\% CI, 22.94-46.90\%]) was also significantly higher than that in short-haired cats $(4 / 64,6.25 \%$ [95\% CI, 2.15$12.28 \%$; $\mathrm{p}<0.001)$ (Table-3). Moreover, there was a significant association between positive Wood's lamp test and dermatophyte-positive culture $(p=0.036$; Table-3). The odds of long-haired cats having 
dermatophytosis were 8.05 (95\% CI, 2.44-33.97) times greater than that in short-haired cats. It should be noted that the number of dermatophytosis-positive domestic short-haired (DSH) cats $(2 / 50,4.00 \%)$ was significantly lower than dermatophytosis-positive Persian $(17 / 47,36.17 \%$; $<<0.001)$ and long-haired mixed breed $(3 / 7,42.86 \% ; p=0.011)$ cats (Table- 3$)$.

The overall sensitivity and specificity of the Wood's lamp test for diagnosing $M$. canis infection were $37.5 \%(95 \% \mathrm{CI}, 21.2-57.3 \%)$ and $96.1 \%(95 \%$ CI, 90.4-98.5\%), respectively (Table-4). The positive and negative predictive values of the Wood's lamp test for diagnosing $M$. canis infection were $69.2 \%$ (95\% CI, 42.4-87.3\%) and 86.8\% (95\% CI, 79.4-91.9\%), respectively (Table-4).

The median hematocrit in cats with dermatophytosis was significantly lower than in cats without dermatophytosis (38.55\% [31.9-53.4\%] vs. 41.5\% [24.2$51.9 \%$ ], $\mathrm{p}=0.0127$; Figure-4). The median (range) of white blood cell count, neutrophil count, and monocyte count in cats with dermatophytosis was significantly higher than those without dermatophytosis (white blood cell count: 10,500 $(4900-20,800)$ cell/ $\mu \mathrm{L}$ vs. $8480(3420-22,400)$ cell $/ \mu \mathrm{L}, \mathrm{p}=0.0014$; neutrophil count: $7096(2646-14,768)$ cell/ $\mu \mathrm{L}$ vs. 5338 (383$15,106)$ cell $/ \mu \mathrm{L}, \mathrm{p}=0.0031$; monocyte count: 334 $(103-1040)$ cell $/ \mu \mathrm{L}$ vs. 184 (0-650) cell $/ \mu \mathrm{L}, \mathrm{p}=0.0005$; (Figure-4). However, the median lymphocyte count and eosinophil count of cats with dermatophytosis did not differ significantly from those in cats without dermatophyte infection (lymphocyte count: 2173 [375-8064] cell $/ \mu \mathrm{L}$ and 2575 [742-7105] cell $/ \mu \mathrm{L}$, $\mathrm{p}=0.1936$; eosinophil count: 531 [197-2288] cell/ $\mu \mathrm{L}$ vs. 481 [62-1885] cell/ $\mu \mathrm{L}, \mathrm{p}=0.0729)$.

The median total protein and albumin levels in cats with dermatophytosis were significantly lower than in cats without dermatophyte infection (total protein: 7.45 [6.2-9.5] g/dL vs. 7.7 [6.4-10.5] g/dL, $\mathrm{p}=0.0055$; albumin: 3.1 [2.2-3.8] g/dL vs. 3.4 [2.5$4.1] \mathrm{g} / \mathrm{dL}, \mathrm{p}<0.0001$; Figure-5). However, the median globulin level in cats with dermatophytosis did not differ significantly from those in cats without dermatophyte infection (globulin: 4.3 [3.6-7.3] g/dL vs. 4.3 [2.8-6.8], p=0.4537; Figure-5). The median blood

Table-1: Demographic distribution of 127 cats enrolled in the present study.

\begin{tabular}{|c|c|c|c|}
\hline Characteristic & Short-haired cats & Long-haired cats & p-value \\
\hline $\mathrm{n}$ & 64 & 63 & - \\
\hline Age (years) & & & 0.8847 \\
\hline Median (range) & $3.2(0.9-13)$ & $3(0.8-11)$ & \\
\hline Sex, \% (n.) & & & 0.1106 \\
\hline Male & $60.9 \%(39)$ & $54.0 \%(34)$ & \\
\hline Female & $39.1 \%(25)$ & $46.0 \%(29)$ & \\
\hline Body weight, kg & & & 0.0131 \\
\hline Median (range) & $4.4(2.4-9.5)$ & $3.8(2.1-10.2)$ & \\
\hline \multicolumn{4}{|l|}{ Breed, \% (n.) } \\
\hline Domestic short haired & $78.1 \%(50)$ & - & - \\
\hline Scottish fold & $7.8 \%(5)$ & - & - \\
\hline American or British short hair & $14.1 \%(9)$ & - & - \\
\hline Persian & - & $74.6 \%(47)$ & - \\
\hline Maine coon & - & $11.1 \%(7)$ & - \\
\hline Mixed breed (hair shaft length $>3 \mathrm{~cm}$ ) & - & $14.3 \%(9)$ & - \\
\hline
\end{tabular}

Table-2: Fungal culture results of samples collected from 127 cats and the association between fungal species and hair length*.

\begin{tabular}{|c|c|c|c|c|}
\hline Fungal species & $\begin{array}{c}\text { Short-haired cats } \\
(n=64)\end{array}$ & $\begin{array}{l}\text { Long-haired cats } \\
\qquad(n=63)\end{array}$ & $\begin{array}{c}\text { Total } \\
(\mathrm{n}=127)\end{array}$ & $\begin{array}{c}\text { Fisher's exact test } \\
\text { ( } p \text {-value) }\end{array}$ \\
\hline \multicolumn{5}{|l|}{ Dermatophyte } \\
\hline Microsporum spp. & 2 & 22 & 24 & $<0.0001$ \\
\hline Trichophyton spp. & 2 & 0 & 2 & 0.0959 \\
\hline \multicolumn{5}{|l|}{ Other fungal species } \\
\hline Aspergillus spp. & 12 & 31 & 43 & 0.0002 \\
\hline Cladosporium spp. & 10 & 2 & 12 & 0.0125 \\
\hline Rhizopus spp. & 2 & 2 & 4 & 0.9872 \\
\hline Absidia spp. & 6 & 2 & 8 & 0.1416 \\
\hline Penicillium spp. & 10 & 2 & 12 & 0.0125 \\
\hline Nigrospora spp. & 2 & 0 & 2 & 0.0959 \\
\hline Cladophialospora spp. & 1 & 1 & 2 & 0.9910 \\
\hline Paecilomyces spp. & 2 & 1 & 3 & 0.5645 \\
\hline Curvularia spp. & 2 & 3 & 5 & 0.6343 \\
\hline Trichoderma spp. & 1 & 2 & 3 & 0.5462 \\
\hline Chaetomium spp. & 2 & 0 & 2 & 0.0959 \\
\hline Bipolaris spp. & 3 & 2 & 5 & 0.6600 \\
\hline
\end{tabular}

*Statistical analysis was compared between short-haired and long-haired cats 
urea nitrogen and creatinine levels in cats with dermatophytosis were significantly lower than in cats without dermatophyte infection (blood urea nitrogen:
$21.5[13-36] \mathrm{mg} / \mathrm{dL}$ vs. 25 [16-39] $\mathrm{mg} / \mathrm{dL}, \mathrm{p}=0.0130$; creatinine: $1.17[0.71-1.72] \mathrm{mg} / \mathrm{dL}$ vs. $1.42[0.96-$ $2.63] \mathrm{mg} / \mathrm{dL}, \mathrm{p}=0.0003$ ); however, both blood urea

Table-3: Dermatophytosis diagnosed by Wood's lamp test and dermatophyte cultures in cats categorized by hair coat length and cat breeds.

\begin{tabular}{|c|c|c|c|c|c|c|}
\hline \multirow[t]{2}{*}{ Cat groups } & \multicolumn{2}{|c|}{ Wood's lamp } & \multirow{2}{*}{$\begin{array}{l}\text { Fisher's } \\
\text { exact-test } \\
\text { (p-value) }\end{array}$} & \multicolumn{2}{|c|}{ Fungal culture dermatophytosis } & \multirow{2}{*}{$\begin{array}{l}\text { Fisher's exact } \\
\text { test ( } p \text {-value) }\end{array}$} \\
\hline & Positive & Negative & & Positive & Negative & \\
\hline \multicolumn{7}{|l|}{ Hair length* } \\
\hline Short-haired cats $(n=64)$ & 3 & 61 & - & 4 & 60 & - \\
\hline Long-haired cats $(n=63)$ & 10 & 53 & 0.044 & 22 & 41 & $<0.001$ \\
\hline \multicolumn{7}{|l|}{ Breed $^{+}$} \\
\hline DSH cats $(n=50)$ & 0 & 50 & - & 2 & 48 & - \\
\hline Scottish fold $(n=5)$ & 1 & 4 & 0.091 & 1 & 4 & 0.253 \\
\hline $\begin{array}{l}\text { American/British short- } \\
\text { haired cats }(n=9)\end{array}$ & 2 & 7 & 0.021 & 1 & 8 & 0.397 \\
\hline Persian $(n=47)$ & 8 & 39 & 0.002 & 17 & 30 & $<0.001$ \\
\hline Maine coon $(n=9)$ & 2 & 7 & 0.021 & 2 & 7 & 0.106 \\
\hline $\begin{array}{l}\text { Mixed breed (hair } \\
\text { length }>3 \mathrm{~cm} ; n=7 \text { ) }\end{array}$ & 0 & 7 & 0.275 & 3 & 4 & 0.011 \\
\hline Summary & 13 & 14 & - & 26 & 101 & $0.036^{\#}$ \\
\hline
\end{tabular}

*Statistical analysis was compared with short-haired cats, ${ }^{+}$statistical analyses were compared with DSH cats, ${ }^{*}$ statistical analysis was compared between Wood's lamp test versus fungal culture

Table-4: Sensitivity, specificity, positive predictive value, negative predictive value, and 95\% CI of Wood's lamp for detecting Microsporum species in short-haired cats, long-haired cats, and total sample.

\begin{tabular}{|c|c|c|c|}
\hline \multirow[t]{2}{*}{ Measurement } & Total cats & Short-haired cats & Long-haired cats \\
\hline & $(n=127)$ & $(n=64)$ & $(n=63)$ \\
\hline Sensitivity (\%; 95\% CI) & $37.5(21.2-57.3)$ & $50(9.5-90.5)$ & $36.4(24.3-47.2)$ \\
\hline Specificity $(\% ; 95 \% \mathrm{CI})$ & $96.1(90.4-98.5)$ & $96.8(89.0-99.1)$ & $95.1(83.9-98.7)$ \\
\hline Positive predictive value $(\% ; 95 \% \mathrm{CI})$ & $69.2(42.4-87.3)$ & $33.3(6.1-79.2)$ & $80.0(49.0-94.3)$ \\
\hline Negative predictive value $(\% ; 95 \% \mathrm{CI})$ & $86.8(79.4-91.9)$ & $98.4(91.3-99.7)$ & $73.6(60.4-83.6)$ \\
\hline
\end{tabular}

CI: Confidence interval
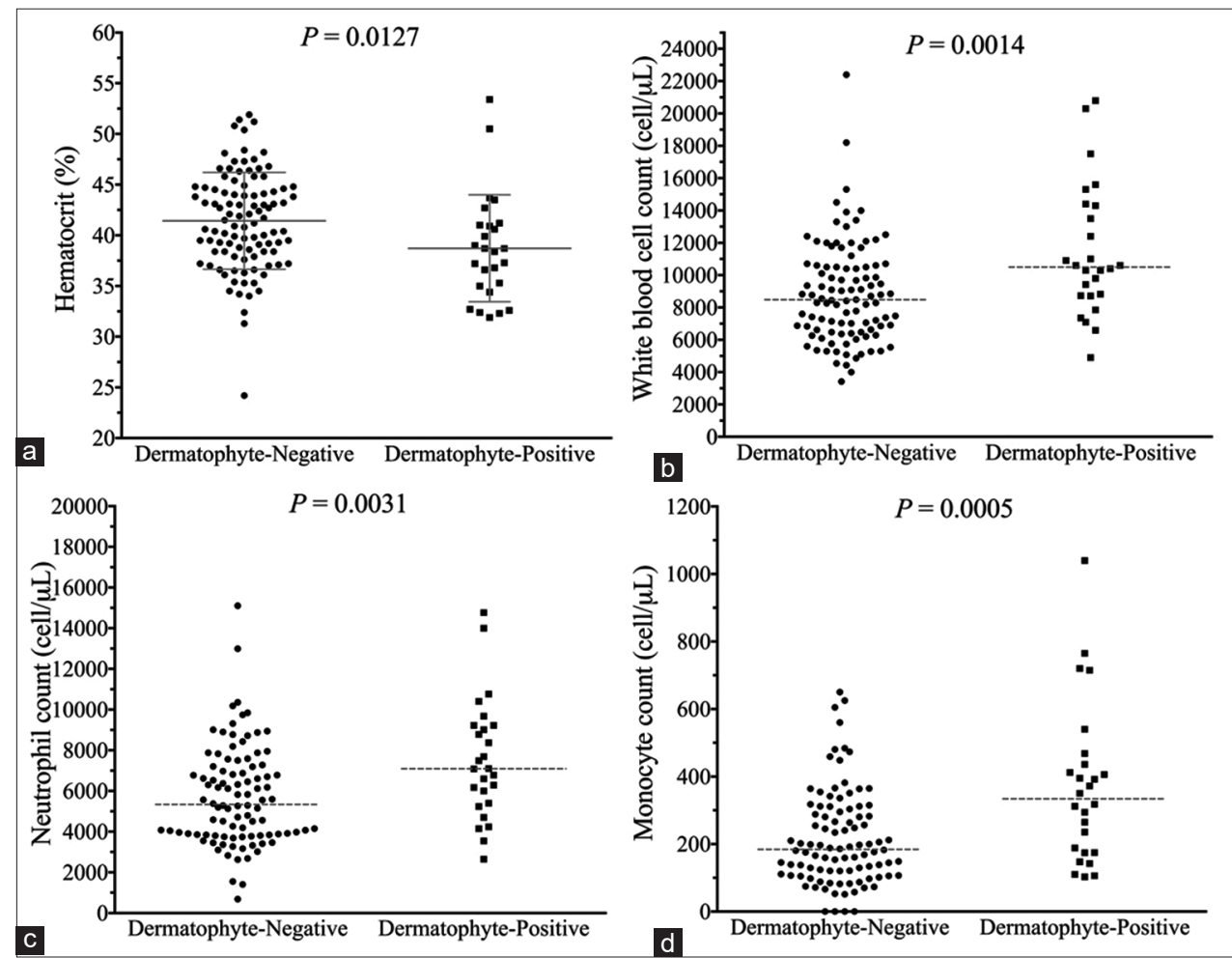

Figure-4: Scatter plots of hematology parameters of cats with negative and positive results for dermatophytosis. (a) Hematocrit, (b) white blood cell count, (c) neutrophil count, and (d) monocyte count. The dashed gray lines represent median. The solid gray lines represent mean and standard deviation. 
nitrogen and creatinine levels were within the normal limits. The median alanine aminotransferase and alkaline phosphatase levels in cats with and without dermatophyte infection were within the normal limits and did not differ significantly (alanine aminotransferase: 55 [21-116] IU/L vs. 53 [25-186] and IU/L, p=0.9600; alkaline phosphatase: 22 [12-67] IU/L vs. 24 [8-88] $\mathrm{IU} / \mathrm{L}, \mathrm{p}=0.6719$; Figure-5).

\section{Discussion}

Dermatophytosis is a common cause of hair loss and scaling in cats, and dermatophytes can transmit to other species, including dogs and humans. In the present study, a survey of healthy short- and long-haired cats was conducted to detect subclinical infection with dermatophytes. Fungal cultures from 26 out of 127 cats $(20.5 \%)$ were positive for dermatophytes, including $M$. canis $(24 / 26,92.3 \%)$ and Trichophyton species $(2 / 26,7.7 \%)$. The prevalence of dermatophyte contamination in long-haired cats $(34.92 \%$; $95 \%$ CI, 22.94$46.9 \%$ ) was significantly higher than that in shorthaired cats $(6.25 \%$; 95\% CI, 2.15-12.28\%), suggesting potential ringworm transmission to humans and animals that contact apparently healthy long-haired or Persian cats. Thus, dermatophyte examinations should be routinely performed due to the higher prevalence of dermatophytosis in long-haired and Persian cats.

Diagnosis of dermatophytosis in the present study was based on the results of fungal cultures using a combination of DTM, ESA, and SDA culture media. A previous study reported the prevalence of dermatophytosis in asymptomatic cats using DTM culture media for dermatophyte isolation [17]. The prevalence ranged from $2.1 \%$ to $15.7 \%$, which was lower than the prevalence identified in the present study (20.5\%) [17]. This discrepancy may due to the combined use of fungal agars, which increases the efficacy of dermatophyte detection. It may also be explained by the different geographic environments of the two studies.

The Wood's lamp test, which uses a longwave ultraviolet light, is a useful screening tool for diagnosing dermatophyte infection on an animal skin [1]. Although the present study indicated a low sensitivity of Wood's lamp for M. canis infection (37.5\% of the infected cats), the test has a very high specificity of $96.1 \%$. Another study reported the positive and negative predictive values of Wood's lamp as $90 \%$ and $94 \%$, respectively [19]. The overall positive predictive value was lower in our study. This may be due to a difference in the clinical dermatology status of the cats from the previous study, which involved testing cats that were clinically suspected of having dermatophyte infection.

Aspergillus species were the most abundant non-dermatophyte fungal species isolated in the present study. Aspergillus species were also previously identified as normal flora in healthy cats [16]. In the present study, Aspergillus species were identified in both short- and long-haired cats. The prevalence of Aspergillus infection in long-haired cats $(49.2 \% ; 95 \%$
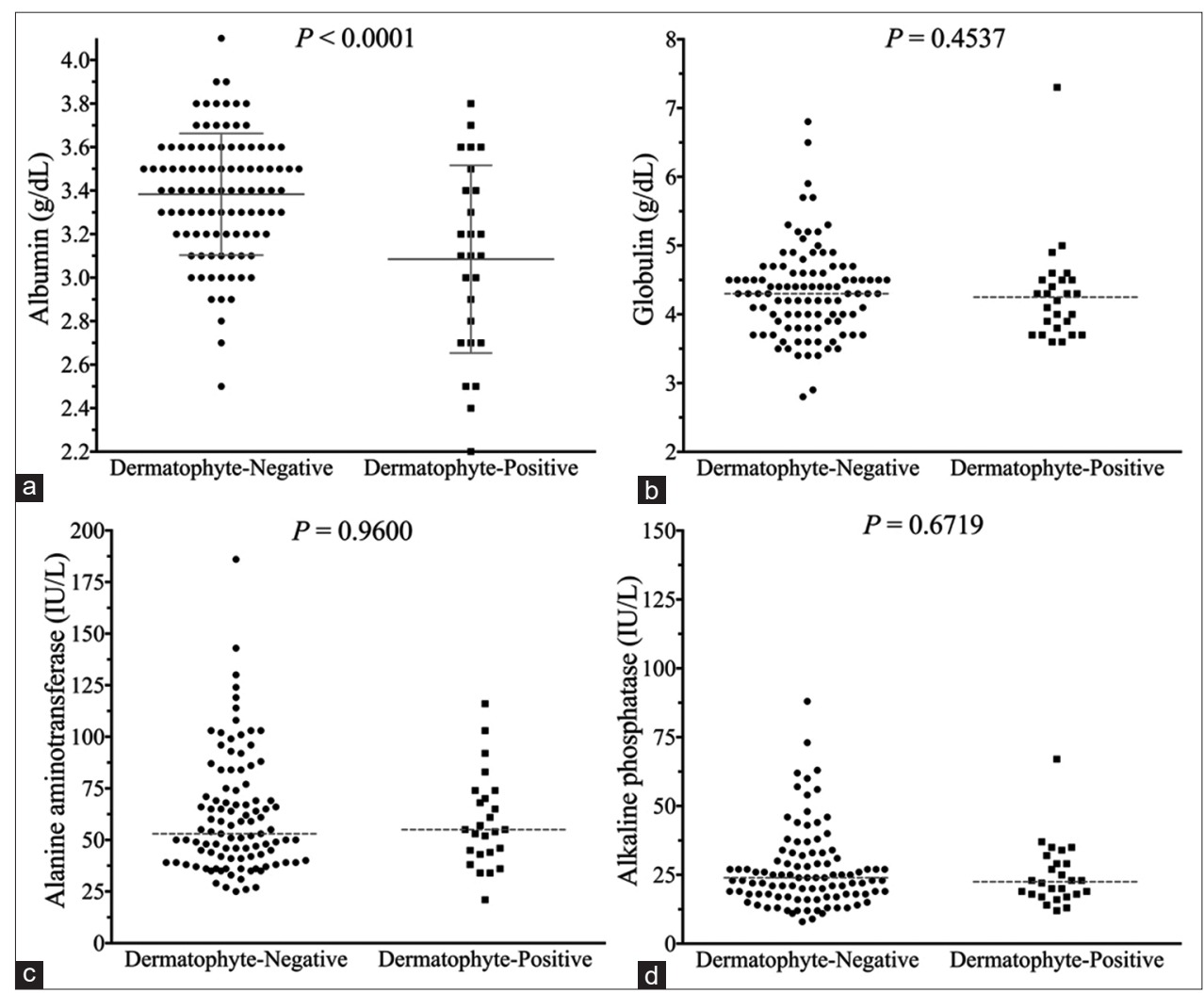

Figure-5: Scatter plots of blood chemistry parameters of cats with and without dermatophytosis. (a) Albumin, (b) globulin, (c) alanine aminotransferase, and (d) alkaline phosphatase. The dashed gray lines represent median. The solid gray lines represent mean and standard deviation. 
CI, 36.38-62.11\%) was significantly higher than that in short-haired cats $(18.75 \%$; 95\% CI, $10.08-30.46 \%$; $\mathrm{p}=0.0002$ ). Our results indicated that the longer and thicker fur of long-haired cats harbored more fungi than that of short-haired cats.

Since the present survey was conducted in FeLVnegative and FIV-negative cats, the results may not reflect the actual prevalence of dermatophytosis in clinical settings. The prevalence of dermatophyte infections in cats infected with FeLV or FIV could be higher due to their compromised immunity and elevated risk of other opportunistic infections [18]. In the present study, Persian cats accounted for $74.6 \%$ of the long-haired cats, which may have skewed the data. Persian cats are known to have a higher susceptibility to dermatophyte fungi infections, and the influence of inheritance of the susceptible genes cannot be ruled out. In the present study, the number of positive dermatophytosis found in Persian cats $(17 / 47,36.17 \%)$ was significantly higher than that found in DSH cats $(2 / 50,4.00 \%)$. Our results were consistent with the findings of a previous study that report that identified dermatophytes in the hair coat of healthy Persian cats, especially from commercial catteries [20]. We also found that cats with dermatophytosis had significantly lower hematocrit and serum albumin levels compared with cats without dermatophytosis, suggesting that infected cats were in poorer health. Leukocytosis with elevated neutrophil and monocyte counts in cats with dermatophytosis may indicate stress or inflammatory response to fungal infections. Therefore, the hematology and blood chemistry profile of cats with dermatophytosis should be monitored.

\section{Conclusion}

The present study revealed that long-haired cats were more susceptible to dermatophyte infection than short-haired cats. Long-haired cats are a potential source of dermatophyte pathogens due to their thicker coat, and fungal infections may spread to other cats and humans. Long-haired cats should be routinely evaluated for dermatophytosis using specific dermatophyte fungal media cultures as well as the Wood's lamp test and blood profiles should be evaluated in cats with dermatophytosis.

\section{Authors' Contributions}

PS: Conceptualization, data curation, formal analysis, funding acquisition, software, writing - original draft. CB and PS: Investigation, methodology, validation, visualization. NT: Project administration and resources and supervision. PS and NT: Writing - original draft. PS, CB, and NT: Writing - review and editing. All authors read and approved the final manuscript.

\section{Acknowledgments}

This study was supported by a grant from the Faculty of Veterinary Medicine, Kasetsart University
(\#62 04), Thailand. The authors thank Hathaipat Rattanathanya, Prakaimook Wimolrachataporn, Suchana Aruvornlop, Pajera Tepsongkroh, and Valeerat Asawakowitkorn for assisting in sample collection. We also thank Miss Orawan Limsivilai and Dr. Chompoonek Yurayart for their technical support on fungal identification. FASTest ${ }^{\circledR}$, FeLV-FIV test kit, and MYKODERMOASSAY TRIO fungal culture plate were provided by MEGACOR Diagnostik $\mathrm{GmbH}$, Austria.

\section{Competing Interests}

The authors declare that they have no competing interests.

\section{Publisher's Note}

Veterinary World remains neutral with regard to jurisdictional claims in published institutional affiliation.

\section{References}

1. Moriello, K.A., Coyner, K. and Paterson, S. (2017) Diagnosis and treatment of dermatophytosis in dogs and cats.: Clinical consensus guidelines of the world association for veterinary dermatology. Vet. Dermatol., 28(3): e266-e268.

2. Frymus, T., Gruffydd-Jones, T., Pennisi, M.G., Addie, D., Belak, S., Boucraut-Baralon, C., Egberink, H., Hartmann, K., Hosie, M.J., Lloret, A., Lutz, H., Marsilio, F., Möstl, K., Radford, A.D., Thiry, E., Truyen, U. and Horzinek, M.C. (2013) Dermatophytosis in cats: ABCD guidelines on prevention and management. J. Feline Med. Surg., 15(7): 598-604.

3. Muller, A., Guaguere, E., Degorce-Rubiales, F. and Bourdoiseau, G. (2011) Dermatophytosis due to Microsporum persicolor: A retrospective study of 16 cases. Can. Vet. J., 52(4): 385-388.

4. Nardoni, S., Mugnaini, L., Papini, R., Fiaschi, M. and Mancianti, F. (2013) Canine and feline dermatophytosis due to Microsporum gypseum: A retrospective study of clinical data and therapy outcome with griseofulvin. J. Mycol. Med., 23(3): 164-167.

5. Sparkes, A.H., Werrett, G., Stokes, C.R. and GruffyddJones, T.J. (1994) Microsporum canis: Inapparent carriage by cats and the viability of arthrospores. J. Small Anim. Pract., 35(8): 397-401.

6. Newbury, S., Moriello, K., Coyner, K., Trimmer, A. and Kunder, D. (2015) Management of endemic Microsporum canis dermatophytosis in an open admission shelter: A field study. J. Feline Med. Surg., 17(4): 342-347.

7. Jacobson, L.S., McIntyre, L. and Mykusz, J. (2018) Comparison of real-time PCR with fungal culture for the diagnosis of Microsporum canis dermatophytosis in shelter cats: A field study. J. Feline Med. Surg., 20(2): 103-107.

8. Bernhardt, A., von Bomhard, W., Antweiler, E. and Tintelnot, K. (2015) Molecular identification of fungal pathogens in nodular skin lesions of cats. Med. Mycol., 53(2): 132-144.

9. Faggi, E., Pini, G., Campisi, E., Bertellini, C., Difonzo, E. and Mancianti, F. (2001) Application of PCR to distinguish common species of dermatophytes. J. Clin. Microbiol., 39(9): 3382-3385.

10. Sierra, P., Guillot, J., Jacob, H., Bussieras, S. and Chermette, R. (2000) Fungal flora on cutaneous and mucosal surfaces of cats infected with feline immunodeficiency virus or feline leukemia virus. Am. J. Vet. Res., 61(2): 158-161.

11. Moriello, K. (2014) Feline dermatophytosis: Aspects 
pertinent to disease management in single and multiple cat situations. J. Feline Med. Surg., 16(5): 419-431.

12. Rezusta, A., de la Fuente, S., Gilaberte, Y., Vidal-Garcia, M., Alcala, L., Lopez-Calleja, A., Ruiz, M.A. and Revillo, M.J. (2016) Evaluation of incubation time for dermatophytes cultures. Mycoses, 59(7): 416-418.

13. Chang, S.C., Liao, J.W., Shyu, C.L., Hsu, W.L. and Wong, M.L. (2011) Dermatophytic pseudomycetomas in four cats. Vet. Dermatol., 22(2): 181-187.

14. Moriello, K.A., Verbrugge, M.J. and Kesting, R.A. (2010) Effects of temperature variations and light exposure on the time to growth of dermatophytes using six different fungal culture media inoculated with laboratory strains and samples obtained from infected cats. J. Feline Med. Surg., 12(12): 988-990.

15. Kaufmann, R., Blum, S.E., Elad, D. and Zur, G. (2016) Comparison between point-of-care dermatophyte test medium and mycology laboratory culture for diagnosis of dermatophytosis in dogs and cats. Vet. Dermatol., 27(4): e284-e368.
16. Meason-Smith, C., Diesel, A., Patterson, A.P., Older, C.E., Johnson, T.J., Mansell, J.M., Suchodolski, J.S. and Hoffmann, A.R. (2017) Characterization of the cutaneous mycobiota in healthy and allergic cats using next-generation sequencing. Vet. Dermatol., 28(1): e71-e117.

17. Mignon, B.R. and Losson, B.J. (1997) Prevalence and characterization of Microsporum canis carriage in cats. J. Med. Vet. Mycol., 35(4): 249-256.

18. Mancianti, F., Giannelli, C., Bendinelli, M. and Poli, A. (1992) Mycological findings in feline immunodeficiency virus-infected cats. J. Med. Vet. Mycol., 30(3): 257-259.

19. Sparkes, A.H., Gruffydd-Jones, T.J., Shaw, S.E., Wright, A.I. and Stokes, C.R. (1993) Epidemiological and diagnostic features of canine and feline dermatophytosis in the United Kingdom from 1956 to 1991. Vet. Rec., 133(3): 57-61.

20. Nitta, C.Y., Daniel, A.G.T., Taborda, C.P., Santana, A.E. and Larsson, C.E. (2016) Isolation of dermatophytes from the hair coat of healthy Persian cats without skin lesions from commercial catteries located in Sao Paulo metropolitan area, Brazil. Acta Sci. Vet., 44(1): 1421.

$* * * * * * * *$ 